\title{
LA DECLARACIÓN UNILATERAL DE INDEPENDENCIA DEL PARLAMENTO DE CATALUÑA EN RELACIÓN CON LA DECLARACIÓN UNILATERAL DE INDEPENDENCIA DE KOSOVO: UNA PERSPECTIVA NO SOLO CONSTITUCIONAL'
}

\author{
The Unilateral Declaration of Independence of the Parliament of Catalonia in \\ relation to the Unilateral Declaration of Independence of Kosovo: not only a \\ constitutional perspective
}

Recibido: 19 de noviembre de 2019

Aceptado: 20 de diciembre de 2019

Andrés Torres Fonseca

Graduado en Filosofía

Graduado en Derecho

andrestorresfonseca37@gmail.com

\section{RESUMEN}

La Declaración Unilateral de Independencia tuvo lugar en Cataluña el 27 de octubre de 2017 y sus consecuencias legales y políticas aún se están dirimiendo. En este trabajo se plantea su similitud o diferencias con la DUI efectuada por Kosovo y la repercusión que tuvo en la comunidad internacional. Que la Corte Internacional de Justicia en su opinión consultiva no prohíba la DUI efectuada por Kosovo ha sido utilizado por el nacionalismo independentista catalán como un argumento válido para reforzar su idea de secesión, que transforman en una secesión unilateral derivada de un derecho de autodeterminación legítimo. En estas páginas, planteo las implicaciones de esta argumentación y las distintas relaciones que se establecen en el caso entre el derecho constitucional y el derecho internacional.

\section{PALABRAS CLAVES}

Declaración unilateral de independencia, procés, autodeterminación, secesión, secesión como remedio.

\begin{abstract}
The Unilateral Declaration of Independence took place in Catalonia on October 27, 2017 and had legal and political consequences that are still being resolved. In this paper, it will be discussed its similarities and differences with the UDI carried out by Kosovo and its effects on the international community. It could be said that the International Court of Justice in its Advisory Opinion does not prohibit the Kosovo UDI and its has been used by Catalan independence nationalism as a valid argument to reinforce its idea of secession, which they transform into a unilateral secession derived from the right of self-determination. In the following pages, the implications of this argument will be outlined, as well as the different relationships established in the case between constitutional law and international law.
\end{abstract}

\section{KEYWORDS}

Unilateral declaration of independence, proces, selfdetermination, secession, remedial secession.

\footnotetext{
${ }^{1}$ Trabajo realizado de acuerdo a la beca concedida en 2018-2019, “Ayudas para la iniciación a la investigación. I Plan Propio de Investigación y Transferencia de la Universidad de Málaga", bajo la dirección de D. Ángel Rodríguez Vergara. Agradecimientos a la Universidad de Málaga y a D. Ángel Rodríguez Vergara.
} 
Sumario: 1. Introducción. 2. Comparativa DUI Kosovo y DUI Cataluña. 2.1. Marco "interno" de Kosovo al efectuar la DUI. 2.2. Marco interno de Cataluña al efectuar la DUI. 3. Autodeterminación y secesión. 3.1. Caso Kosovo desde la secesión como remedio. 3.2. Caso Cataluña desde la secesión como remedio. 4. Conclusiones. Bibliografía.

\section{Introducción.}

A raíz de la Declaración Unilateral de Independencia (en adelante, DUI) aprobada por el Parlament de Cataluña el 27 de octubre de 2017 el nacionalismo catalán ve cumplido sus objetivos. El de este artículo será comprobar si esta declaración puede ser aceptada bajo el derecho constitucional. Con esa intención, se relaciona con la DUI efectuada por la Asamblea de Kosovo en 2008 y la Opinión consultiva de la Corte Internacional de Justicia (en adelante, CIJ), que no prohibiría la DUI desde el derecho internacional, lo que desde el nacionalismo catalán se entenderá que legitima su propia DUI y permitiría ampararla en el derecho de autodeterminación reconocido por la comunidad internacional. La relación entre derecho constitucional y derecho internacional público es constante en este artículo dado el tema elegido, en él establezco la oportuna distinción de tratar la autodeterminación desde el derecho internacional y la secesión bajo la perspectiva del derecho constitucional.

Para ello esbozo el procés y lo que significa para consensuar y efectuar la DUI, a través del relato del agravio y la recreación de la identidad nacional catalana. Se compara el contexto previo a la DUI en Cataluña con el de Kosovo. Luego se analiza la Opinión consultiva de la CIJ y porqué consideran que la DUI de Kosovo no estaría prohibida por el derecho internacional. Se exponen los elementos más relevantes para llegar a esta conclusión y se comprueba que en el caso de Cataluña se puedan asimilar y comprobar que la agenda nacionalista no esté equivocada al internacionalizar el procés, para lograr su aceptación.

Una vez que el procés cobra total relevancia internacional gracias a la DUI catalana, es necesario aclarar los conceptos de, autodeterminación, secesión y secesión como remedio, y las distintas relaciones que se establecen entre ellos. En este punto analizo si el caso de Cataluña es aceptado bajo la doctrina de la secesión como remedio, tomando como referencia la solución que obtiene el caso de Kosovo.

Hacer partícipes a la comunidad internacional de una secesión ilegítima es fundamental para los grupos nacionalistas por lo que en ambas situaciones se defenderán postulados semejantes sobre la necesidad del planteamiento de una solución política, ya que han abandonado la vía de la legalidad al pretender una ruptura con el marco constitucional. Esta internacionalización del procés a través de la DUI será clave en todo el artículo y se plantea como eje central del mismo.

Por último, a través de la comparación con los dos casos tras el recorrido de la unilateralidad, considero si pudiera aceptarse la DUI desde un derecho interno que no la contemple y si para ello fuera necesario la opinión favorable de la comunidad internacional. O, por el contrario, aunque la DUI no contara con la aprobación de la comunidad internacional, si al comprobar una situación excepcional pudieran darle una solución política que satisfaga a las partes implicadas. 


\section{Comparativa DUI Kosovo y DUI Cataluña.}

Entre la declaración unilateral de independencia efectuada en la Asamblea de Kosovo el 17 de febrero de $2008^{2}$ y la DUI realizada por el presidente de la Generalitat, Sr. Puigdemont, el 10 de octubre de 2017 en el Parlament ${ }^{3}$, aprobada por el Parlament el 27 de octubre de 2017, hay una Opinión consultiva de la Corte Internacional de Justicia ${ }^{4}$ que, según la tesis esgrimida por el independentismo catalán, avalaría ambas desde el derecho internacional. El propósito de este epígrafe es analizar el dictamen de la CIJ y comprobar si realmente aprueba desde el derecho internacional una declaración unilateral de independencia y las razones que para ello emplea. Además, se cuenta con la opinión separada de los magistrados que votaron en contra de este dictamen y las cuestiones que plantearon a la mayoría. Una vez se verifique esta intencionalidad de la CIJ de no prohibir la DUI de Kosovo al no violar el derecho internacional (diez votos contra cuatro) la comparo con la DUI catalana y los distintos puntos que puedan tener en común.

El resurgir del nacionalismo en distintas regiones europeas, con reivindicaciones históricas, ha brotado con fuerza. Es un hecho manifiesto que el nacionalismo catalán se ha apropiado de la Opinión consultiva de la Corte Internacional de Justicia sobre la declaración unilateral de independencia de Kosovo para reafirmarse en sus ideas y fortalecer un relato independentista dentro de Cataluña. Siempre apuntando a la comunidad internacional, para validar una opinión que admita desde el derecho internacional la declaración unilateral de independencia. Lo que en la lógica secesionista implica que una vez aceptada y reconocida la declaración de independencia ésta tendría que ser aceptada por el derecho constitucional español.

\subsection{Marco "interno" de Kosovo al efectuar la DUI.}

Se inicia el análisis del dictamen de la Corte en este epígrafe para comprobar la opinión de los magistrados al respecto y el marco interno legal en el momento de producirse la declaración. Se expone primero, el marco interno en Kosovo durante la DUI, luego el de Cataluña.

Puntualizo que no trato el marco constitucional de Serbia aplicable a Kosovo ya que la Resolución $1244(1999)^{5}$ lo deslegitima e impone uno propio, internacional. Así, la

\footnotetext{
${ }^{2}$ Ver la noticia de la declaración disponible en:

https://www.elmundo.es/elmundo/2008/02/17/internacional/1203250080.html [consultada el 25 de marzo de 2019].

El texto íntegro de la DUI Kosovo traducido al español disponible en:

https://e00elmundo.uecdn.es/documentos/2008/02/17/independencia_kosovo.pdf No hay fuente jurídica de la publicación de la DUI ya que la efectuaron personas al margen de la Asamblea de Kosovo, se analizará en el siguiente epígrafe.

${ }^{3}$ Ver la noticia de la declaración disponible en:

https://elpais.com/ccaa/2017/10/10/catalunya/1507624143_410500.html [consultada el 25 de marzo de 2019].

El texto íntegro de la DUI catalana disponible en:

https://www.lavanguardia.com/politica/20171010/431970027817/declaracion-de-independencia-

catalunya.html. No se encuentra publicada en el Boletín Oficial del Parlamento de Cataluña al ser simbólica según sus propios autores, como expondré más adelante.

4 International Court of Justice. Accordance with International Law of the Unilateral Declaration of Independence in Respect of Kosovo, Advisory Opinion, 2010 I.C.J. 404 (July 22). Disponible en:

https://www.icj-cij.org/files/case-related/141/141-20100722-ADV-01-00-EN.pdf (Utilizo la versión en español).

${ }^{5}$ Aprobada por el Consejo de Seguridad de las Naciones Unidas el 10 de junio de 1999.
} 
Corte defiende que el marco interno internacional puede respaldar la DUI ya que desde la vertiente provisional tenía como objetivo principal obtener un gobierno estable en Kosovo. Si para obtener esta estabilidad era necesario vulnerar la Resolución internacional mediante la DUI es aceptable en cierto modo por la CIJ, cuando incorporan el argumento de "solución política".

Primero, atiendo a la argumentación de la Corte para adecuar la DUI al marco constitucional interno creado por la UMNIK y la Resolución 1244 (1999). De nuevo los magistrados se contradicen ya que indican que el derecho constitucional interno rige el acto de hacer ese tipo de declaraciones de carácter político, mientras que la competencia de la Corte para emitir una Opinión consultiva se limita a cuestiones de derecho internacional. ${ }^{6}$ Sin embargo, los magistrados recurren al derecho interno en el momento de la DUI de Kosovo para comprobar que era conforme a él, cuando antes lo niegan y declaran que únicamente acuden al derecho internacional. Entiendo que, aunque sea un orden jurídico interno que proviene de una Resolución emitida por un órgano internacional a lo largo de toda la Opinión consultiva se considera como marco constitucional, por lo que o no deberían pronunciarse sobre este punto, o en todo caso no admitir la DUI desde el marco interno.

Los magistrados no mencionan en este apartado nada de lo anterior e inician una nueva argumentación que gira en torno a establecer el estatuto definitivo de Kosovo para adecuar la DUI. Se coloca la premisa de gobierno estable de Kosovo y su estatuto definitivo sobre la mesa y legitimarlo a cualquier precio parece la finalidad de la Corte. Este era el objetivo final de la Resolución 1244 (1999) del Consejo de Seguridad, crear un régimen provisional para Kosovo y así a encauzar el proceso político a largo plazo. El problema es que la Resolución no contenía ninguna disposición que se ocupara del estatuto definitivo de Kosovo o de las condiciones para alcanzarlo. ${ }^{7}$

La Corte alude a otros casos (Chipre) donde si se establecen condiciones específicas para acordar el estatuto permanente el Consejo de Seguridad lo exige restrictivamente, y se especifican en la Resolución pertinente. ${ }^{8}$

En cambio, del tenor de la Resolución 1244 (1999) se desprende que el Consejo de Seguridad no se reservó la decisión definitiva sobre la situación en Kosovo y que guardó silencio sobre las condiciones para el estatuto definitivo de Kosovo. Por lo tanto, afirman que esta Resolución no impedía la adopción de la declaración de independencia de 17 de febrero de 2008 porque ambos instrumentos operaban a distinto nivel: a diferencia de la Resolución 1244 (1999), la declaración de independencia era un intento de fijar de manera definitiva el estatuto de Kosovo. ${ }^{9}$

La Corte reafirma con la interpretación de la Resolución 1244 (1999) que el objeto y fin de la Resolución es establecer una administración provisional para Kosovo sin adoptar ninguna decisión sobre su estatuto definitivo ${ }^{10}$, lo que permite que la DUI no esté prohibida bajo este marco interno tratándose de una Resolución internacional. ${ }^{11}$

\footnotetext{
${ }^{6}$ Párrafos 26 y 27 de la Opinión consultiva de la CIJ.

${ }^{7}$ Párrafo 114 de la Opinión consultiva de la CIJ.

${ }^{8}$ Párrafos 115, 116 de la Opinión consultiva de la CIJ.

${ }^{9}$ Párrafo 114 de la Opinión consultiva de la CIJ.

${ }^{10}$ Cardona, J. (2011): "La relevancia (o no) de la administración internacional de Kosovo en la opinión consultiva de la Corte Internacional de Justicia de 22 de julio de 2010: perplejidades de un lector," Revista Española de Derecho Internacional, Núm. 63, p. 83.

11 Párrafo 118 de la Opinión consultiva de la CIJ.
} 
Es en este párrafo donde explican la "solución política" como fin último de la Resolución internacional para dar por finalizado el conflicto kosovar con Serbia. Esta idea se menciona en la Resolución 1244 (1999) como fin de la presencia internacional en Kosovo, para conseguir una solución política ayudando al autogobierno de Kosovo. Esta interpretación extensiva de solución política realizada por la Corte permite a los magistrados considerar que la Resolución internacional no limita ni prohíbe, y otorga cualquier tipo de herramientas legales o políticas a los sujetos legitimados para ello si buscan una solución. De nuevo, siguiendo los razonamientos anteriores que manifiestan si la Resolución 1244 (1999) prohibía la DUI, la Corte establece que no, ya que no hay una prohibición específica de declarar la independencia de Kosovo respecto de la República de Serbia por parte de actores políticos. Por lo tanto, la DUI no vulnera la Resolución 1244 (1999), marco interno aplicable en ese momento en Kosovo. ${ }^{12}$

Además de no vulnerar el marco interno desde la Resolución internacional, la Corte también se pronuncia afirmando que tampoco contradice el marco constitucional de Kosovo establecido bajo la UMNIK. La UMNIK creaba y consolidaba las instituciones provisionales de autogobierno de Kosovo. Estas instituciones no podían declarar la independencia unilateral ya que excedía sus competencias y no venía recogido en este marco constitucional. Para eludir esto la CIJ recuerda que la declaración de independencia no fue efectuada por las instituciones provisionales de Kosovo sino por actores no vinculados a estas instituciones. Volviendo a la identidad de los autores y su no vinculación al gobierno provisional (que sí lo estaban) ${ }^{13}$ los magistrados consideran que al ser un acto que no pretendía surtir efectos y sólo buscar soluciones no está recogido como competencia de las autoridades de Kosovo, por lo que tampoco se vulnera este marco constitucional. ${ }^{14}$

La DUI efectuada en Kosovo en 2008 no vulnera de ningún modo para la CIJ el marco jurídico interno de Serbia, ni de Kosovo. En primer lugar, consideran el marco interno de Kosovo propio, desvinculado de la Constitución Serbia, impuesto por una Resolución internacional, la Resolución 1244 (1999). Aludiendo a los objetivos finales de esta Resolución como el conseguir un gobierno estable para Kosovo pretendiendo una solución política justifican la DUI. Si el marco interno, proporcionado por una Resolución internacional, tiene como fin la solución política, la DUI entienden recae bajo esta interpretación de solución política, por lo que la Resolución 1244 (1999) no la prohibiría, lo que conlleva a que la DUI no vulnere el marco interno. Además, en segundo lugar, la UMNIK también consolida el marco interno dando legitimidad a las instituciones de autogobierno provisionales en Kosovo. Parece claro que no está entre sus competencias efectuar una declaración unilateral de independencia, pues bien, para no violar este marco interno propuesto por la UMNIK, la Corte considera y repite el argumento de la identidad de los actores. Al ser actores políticos su declaración no tiene fuerza para vincular a la UMNIK y las instituciones de autogobierno así creadas, por lo que tampoco la DUI vulneraba el marco interno en este sentido.

Una vez se expone como la CIJ no considera que la DUI de Kosovo vulnere el marco interno ni desde la Resolución 1244 (1999) ni desde el marco constitucional provisional creado por la UMNIK, voy a comprobar si la DUI catalana estaría admitida por el marco constitucional español.

\footnotetext{
12 Párrafo 119 de la Opinión consultiva de la CIJ.

${ }^{13}$ Cardona, J. (2011): op. cit., p. 93.

${ }^{14}$ Párrafos 120-121 de la Opinión consultiva de la CIJ.
} 


\subsection{Marco interno de Cataluña al efectuar la DUI.}

La DUI catalana se efectúa bajo el marco constitucional español que de ninguna manera contempla la secesión unilateral, ni otorga a una Comunidad Autónoma la legitimidad para declarar la independencia unilateral. No se ajusta de ningún modo al ordenamiento jurídico español. Las Leyes de Transitoriedad jurídica y Fundacional de la República $(19 / 2017,20 / 2017)^{15}$, aprobadas en los Plenos del Parlament el 6 y 7 de septiembre al margen del procedimiento exigido por la ley, y que marcan el camino para que desde el pretendido referéndum del 1 de octubre la soberanía del pueblo catalán se materialice en la DUI fueron declaradas nulas por el Tribunal Constitucional. ${ }^{16}$

La soberanía del pueblo catalán no podía expresarse en el referéndum ya que no se contempla en el marco interno español la figura del referéndum para este tipo de consultas, ${ }^{17}$ por lo que del mandato de las urnas del 1-O no se podía inferir que el pueblo catalán quería la independencia unilateral y habilitaban para ello a sus representantes políticos.

La DUI excedía las funciones del Parlamento catalán, vulnerando el marco constitucional, aunque atendiendo a la Opinión consultiva de la CIJ se puede pensar que al efectuarse por unos políticos que carecen de la fuerza necesaria para hacerla efectiva se debería tratar como un acto político sin efecto alguno, con lo que los actores no se responsabilizarían de la DUI. Está opinión de la Corte pudo ser el germen que impulsó la consideración de la DUI por los políticos secesionistas como una aceptación favorable a sus pretensiones. Lo dejan claro en la ruta a seguir dentro del marco constitucional con el Libro Blanco de la Transición Nacional de Cataluña, que desembocaría en una posible declaración unilateral. Esta obra recoge los distintos informes dictados por expertos en diversos ámbitos que desde el Consejo Asesor para la Transición Nacional (CATN), que aconsejan al Govern la trayectoria para alcanzar la independencia con la DUI como último recurso.

Este órgano, el CATN, instituto político creado por la Generalitat en 2013, abordaba desde el marco interno español los diferentes escenarios que podrían producirse a raíz de la DUI. En primer lugar, defienden que estaría aceptada por el derecho internacional y que si bien el caso de Cataluña no es Kosovo y no hay una intervención internacional habría que atender a una interpretación flexible, y entender que la Opinión consultiva propone incluir otros contextos más modernos que amplíen los límites de la autodeterminación. Siguiendo con esta argumentación indican que el caso de Cataluña va más allá y por lo tanto encaja con una opinión favorable a la DUI, y no rompería con el marco interno español. ${ }^{18}$ Los motivos que aprecian para concluir que el caso catalán va más allá de Kosovo únicamente se mencionan como una interpretación actualizada del caso acorde con el contexto político catalán bajo la autoridad central española.

Esto facilita que el grupo catalán secesionista entienda que la DUI catalana será aceptada por el derecho internacional. Frente a una aceptación de la DUI por el derecho

\footnotetext{
${ }^{15}$ Publicadas en el Boletín Oficial del Parlamento de Cataluña, XI Legislatura, n. ${ }^{\circ}$ 500, de 6 de septiembre de 2017 y en el Boletín Oficial del Parlamento de Cataluña, n. ${ }^{\circ}$ 507, de 7 de septiembre de 2017.

${ }^{16}$ Ver la respuesta del Tribunal Constitucional a las "leyes de desconexión" en la STC 124/2017 de 8 de noviembre de 2017 que declara nulas e inconstitucionales dichas resoluciones del Parlament.

17 En relación al referéndum que habilitaría las "leyes de desconexión" el Tribunal Constitución se manifiesta en la STC 117/2017 de 17 de octubre de 2017, declarándolo nulo e inconstitucional.

18 V.V. A.A. (2014): Libro Blanco de la Transición de Cataluña, Barcelona: Generalitat Catalunya. Departamento de Presidencia, p. 21.
} 
internacional como salvoconducto para vulnerar el marco interno y no obtener ningún reproche por parte de la autoridad central, también se mencionan otros mecanismos que desde el derecho interno se opondrían a la DUI.

Siendo la aprobación de la DUI, de carácter instrumental y político, en sede parlamentaria mediante la vía de urgencia y pretendiendo una mayoría de votos no especificada, según sus idearios, se planteaban diversas opciones por parte del grupo secesionista catalán que pudieran contrarrestarla desde el marco interno.

En segundo lugar, desde el marco constitucional aludían que el estado español podía recurrir al art. 161.2 CE y al art. 155 CE, para obtener la suspensión automática de la autonomía catalana, según el Libro Blanco. ${ }^{19}$ Incluso llegan más allá en sus elucubraciones y plantean un hipotético estado de excepción y de sitio, regulado en la LO 4/1981. Para justificar la respuesta del estado a la DUI desde estos mecanismos consideran que únicamente sería en el supuesto que la proclamación de la independencia unilateral estaba alterando gravemente el normal funcionamiento de las instituciones democráticas y constituyera un acto de fuerza. Consideraban que la DUI no iba a causar este problema, ni se llevaría a cabo mediante la fuerza, por lo que tampoco responderían desde el derecho penal. Establecieron que hacer efectiva la DUI no implicaba recaer en el supuesto de rebelión, ya que la violencia sería inexistente. La sedición tampoco podría aplicársele, ya que, aunque la DUI pretendía un régimen de autogobierno caracterizado por inaplicación de leyes o el incumplimiento de determinados acuerdos o de resoluciones administrativas o judiciales, no parece, en cambio, que estuviera el requisito de proceder tumultuariamente. ${ }^{20}$

Al margen de las posibles consecuencias de la DUI según el CATN, lo que ocurrió fue lo siguiente: tras votar la DUI, y aprobarla en el Parlament, ésta primero fue suspendida y luego declarada inconstitucional por el TC. También se aprobó el art. 155 $\mathrm{CE}$ en Cataluña por el Senado, y se aplicó por el Gobierno español. Algunos líderes del procés, en prisión provisional, se hallaban inmersos en un proceso judicial para dirimir su posible responsabilidad penal. Al respecto de esto último el Tribunal Supremo ya ha considerado que sí hay responsabilidad penal en los hechos. ${ }^{21}$ Uno de los autores de la DUI no quiso enfrentarse a esta responsabilidad penal y abandonó el Estado español, a día de la elaboración de este artículo continúa fugado.

Se cumple, en cierto modo, el camino esbozado por el Libro Blanco, aunque con consecuencias no esperadas para sus actores. Tras la respuesta del estado español se demuestra que la DUI vulneró el marco jurídico interno y de ningún modo estaba amparada bajo la Constitución como hecho extraordinario político, o situación irremediable que pudiera cobijarse bajo el derecho internacional. El relato del agravio con los sucesos del 1-O como máxima expresión no convence para internacionalizar el proceso y legitimar la secesión.

De acuerdo con la comparación que se sigue a lo largo de este apartado, si la DUI no se permite desde el marco interno, la única razón para considerar que no vulnera el marco constitucional sería la identidad de los autores. Si bien, en un principio la DUI se proclama y se firma en un espacio no habilitado para ello, posteriormente se vota y se aprueba en el Parlament en condiciones irregulares. La votación secreta por llamada

\footnotetext{
${ }^{19}$ Ibíd., p. 27.

${ }^{20}$ Bárcena, J. (2016): "Manual para construir un estado (Los informes del Consejo Asesor para la Transición Nacional de Cataluña)", Teoría y Realidad Constitucional, Núm. 37, p. 510.

${ }^{21}$ Ver la sentencia del Tribunal Supremo STS 459/2019 de 14 de octubre de 2019.
} 
nominal en urna, con la advertencia de los letrados de la cámara de su ilegalidad al estar suspendida por el TC, con la mitad de la cámara ausente en ese momento, y un resultado de 70 votos a favor en un órgano compuesto por 135 miembros. Esto hace cuestionarse la propia validez y fuerza de la DUI desde sus propios autores. No proclaman la DUI dentro del marco constitucional rompiéndolo, sino que el artificio para llevarla a cabo escapa al procedimiento de aprobación de cualquier resolución parlamentaria. Parece que en cierto modo si fue un mero acto político sin ninguna trascendencia dentro del marco interno, y como acto político tampoco sería cuestión de debate por la Corte Internacional de Justicia.

Considero que la falta de legitimidad de los autores, al exceder de sus funciones permitidas por ley, y pretender aprobarla mediante un procedimiento no adecuado para ello, hace que la DUI carezca de ninguna fuerza vinculante y su verdadera cualidad es la de ser un instrumento político. No sería la DUI el final del procés, sino continuar el procés desde la DUI para que lo proyecte hacia una atención internacional más relevante.

Así, el objetivo de la DUI, como acción política sería que desemboque en una atención internacional que asentara las bases de un proceso secesionista con trascendencia fuera del estado español. Esto legitimaría la secesión a través de una hipotética secesión como remedio, como expondré en el siguiente epígrafe. Me lleva a la siguiente dimensión del procés donde a través de la DUI cobran pleno sentido términos como autodeterminación y secesión como remedio, buscando una aceptación de la comunidad internacional y una internacionalización de un proceso secesionista que en un principio pertenecía exclusivamente al marco interno.

Desde el inicio del procés y tal y como se manifiesta en sus antecedentes directos buscar esta vertiente internacional ha sido obligatoria para el grupo secesionista, y con la proclamación de la DUI se consuma este propósito.

\section{Autodeterminación y secesión.}

En este epígrafe delimitaré los conceptos de autodeterminación y secesión para dejar claras las implicaciones del artículo en derecho internacional y derecho constitucional, ya que aludo en numerosas ocasiones a las relaciones establecidas entre dichas ideas. Tal y como se verá ambos términos se hayan entrelazados y es difícil desligarlos en el tema que nos ocupa. Siendo estos conceptos imprescindibles en el caso de Kosovo, un conflicto reconocido y con implicaciones por parte de la comunidad internacional; y en el caso de Cataluña, conocido internacionalmente y con aspiraciones por parte de sus actores a esta implicación. ${ }^{22}$

Hay que considerar el derecho de autodeterminación de los pueblos desde el derecho internacional como el principio de un grupo nacional para reivindicar y elegir por ellos mismos su organización política y relacionarse con otros grupos. Término difuso y complejo que goza de numerosas aproximaciones desde multitud de autores. ${ }^{23}$

En este apartado la entiendo como el derecho de las minorías de una región, en un principio, coloniales, para reforzar su proyección internacional y su plena relación con otros estados a través de un intento, que se puede materializar, de corresponderse como

\footnotetext{
${ }^{22}$ Molina, I. (2018): "La dimensión internacional y europea del procés", en Coll, J., Molina, I. y Maldonado, M. (ed.) Anatomía del procés. Barcelona: Debate, p. 201-220.

23 Ver entre otros: Buchheit, L.C. (1978): Secession: the legitimacy of self-determination, New Haven: Yale University Press; Knop, K. (2002): Diversity and Self-Determination in International Law. Cambridge: Cambridge University Press.
} 
un estado libre y soberano, y así desarrollar su estatus político y económico social. ${ }^{24}$ Puede identificarse en el caso del que me ocupo con el derecho a decidir como fin último del procés y término fundamental en la cuestión catalana. Consistiría este derecho a decidir en la capacidad de un grupo, dentro de una región, para reivindicar su estatus político en lo que se refiere a su mantenimiento como parte integrante del estado del que forma parte $y$, en su caso, finalizar la configuración de su estatuto político en el seno de aquel. Una capacidad que incluye la posibilidad de decidir la independencia, aunque no sea en absoluto este el fin último de la autodeterminación. ${ }^{25}$ Una pretensión que trata de romper con el marco constitucional y la soberanía del estado anterior, o determinar el estatus político de esa comunidad dentro del estado en el supuesto de que optase por mantenerse en su seno. ${ }^{26}$

Estas minorías consideradas aquí como grupo comparten en algunos casos rasgos identitarios, que desembocan en nacionalismos, tales como lenguaje, religión o etnia. Así, el principio de autodeterminación de la minoría estatal lleva configurándose como principio general a partir de la I Guerra Mundial $^{27}$ y desarrollándose tras la II Guerra Mundial, recogiéndose en los textos internacionales. Una de las primeras menciones en estos textos, es en la Carta de San Francisco, siguiendo con la Resolución 1514 (XV) ${ }^{28}$ y los Pactos de Derechos Civiles y Políticos, y de Derechos Económicos, Sociales y Culturales de 1966, tratando la autodeterminación de los grupos coloniales u ocupados que reivindican su soberanía. Sigue con la Resolución $2625(\mathrm{XXV})^{29}$ que completa lo anterior con el inicio del reconocimiento de la doctrina internacional que avalaría la autodeterminación más allá de los casos coloniales, presentando así el derecho a la secesión como remedio de las minorías o grupos étnicos cuando peligren sus derechos. Si mencionamos el párrafo sexto de la Resolución 1514 (XV), que afirma que "todo intento encaminado a quebrantar total o parcialmente la unidad nacional y la integridad territorial de un país es incompatible con los propósitos y principios de la Carta de las Naciones Unidas" ${ }^{30}$, se observa cómo, en principio, el derecho internacional no fomenta el reconocimiento de nuevos estados.

Partiendo de esta base hay que completar que el derecho internacional reconoce el derecho de autodeterminación en su dimensión interna (derecho al gobierno democrático) a todos los pueblos, pero lo limita en su vertiente externa (derecho a decidir el estatus externo del territorio) a los pueblos de un territorio dependiente, contexto colonial, donde aún no se ha constituido un estado, permitiendo una opción a la creación de nuevos estados. ${ }^{31}$ Esta limitación en su vertiente externa cambia a partir de la introducción de la doctrina internacional que avala la secesión como remedio. Tal y como queda expuesto en los textos internacionales la pretensión de autodeterminación está amparada por el derecho internacional, en primer lugar, siempre que provenga desde una colonia, protegiendo un proceso de descolonización; rechazando una ocupación extranjera; o

\footnotetext{
${ }^{24}$ Martínez, A. (2015): El derecho de autodeterminación de los pueblos en el siglo XXI. Navarra: Thomson Reuters Aranzadi, p.127.

${ }^{25}$ López -Basaguren, A. (2016): "Demanda de secesión en Cataluña y sistema democrático. El procés a la luz de la experiencia comparada", Teoría y Realidad Constitucional, Núm. 37, p. 165.

${ }^{26}$ Ibíd., p. 167.

${ }^{27}$ Ibíd., p. 168.

${ }^{28}$ Aprobada por la Asamblea General de las Naciones Unidas el 14 de diciembre de 1960.

${ }^{29}$ Aprobada por la Asamblea General de las Naciones Unidas el 24 de octubre de 1970.

${ }^{30}$ López -Basaguren, A. (2016): óp. cit., p. 169.

${ }^{31}$ Soroeta, J. (2018) "Quo Vadis Cataluña? La unilateralidad, un viaje de corto recorrido", Anuario Español de Derecho Internacional, Núm. 34, pp. 323-342.
} 
desde la opresión de un régimen racista que dañe los derechos humanos de las minorías. ${ }^{32}$ Estos últimos casos serían los conocidos en el supuesto de secesión como remedio, como la última solución de un territorio, inmerso en una crisis territorial, que quiere defender su soberanía ya que peligran los derechos humanos y civiles de las minorías. En principio, se esboza la secesión como remedio como el mecanismo último para defender los derechos de las minorías frente a la opresión del estado y permitir que se defiendan de dicho estado ejerciendo su derecho excepcional recogido en la secesión como remedio.

Aceptado el derecho de autodeterminación desde sus dos vertientes (interna y externa) se puede encontrar un proceso de independencia amparado bajo el principio de libre determinación recogido en el derecho internacional. Pero no siempre se obtienen respuestas idénticas desde la comunidad internacional a situaciones a priori semejantes, como analizaré, ya que en algunas ocasiones la respuesta es no romper la unidad nacional, aunque puedan parecer casos de la secesión como remedio.

Tras el breve repaso al principio de autodeterminación de los pueblos es necesario comprobar en qué medida la secesión está ligada a la autonomía de una región y su derecho a decidir. Antes delimitaré la secesión para seguir sus implicaciones a lo largo del epígrafe adecuándose a los casos concretos que presento.

Primero, presento la secesión como un concepto difícil de acotar, y confundido en ocasiones con términos parecidos como revolución, rebelión, o desobediencia civil. Ideas que pueden encontrarse en el proceso secesionista, pero no podemos reducir este a las situaciones mencionadas. Segundo, se acepta en principio la secesión de un territorio como la ruptura con el marco constitucional vigente, contemplado por el propio texto constitucional, para, posteriormente, configurar uno nuevo que se adecúe a las pretensiones políticas de la región secesionista. Tercero, el objetivo de la secesión no trata de derrocar el gobierno existente ni cree cambiar el orden constitucional para adecuarlo a sus objetivos económicos o sociopolíticos, sino limitar el poder del estado dentro del territorio que ocupa. Propone no estar restringido por el orden interno creado ya en el estado. $^{33}$

El proceso secesionista reduce el poder del estado en su territorio para fortalecer su propio grupo dentro de una región en concreto. Los integrantes de este grupo limitan al máximo el poder estatal mientras van ampliando su autonomía. Para ello no necesitan la eliminación del gobierno central ni negar la autoridad política de éste, tan sólo reaccionan frente a esta autoridad central manifestando que no tiene ninguna ascendencia sobre su grupo ni sobre su territorio. Por lo tanto, tampoco el fin último de la secesión es la independencia ya que el proceso secesionista puede iniciarse para no reconocer el marco interno vigente de un estado en concreto, pero aceptar reconocer una autoridad distinta sobre su territorio no configurada por ellos, es decir, no constituirse en un estado soberano sino unirse a uno ya existente. ${ }^{34}$ Aunque los casos de los que me ocupo sí tienen la independencia como fin último y esperado al iniciar la secesión y no quieren integrarse en ninguna otra autoridad que no sea la conseguida por su propio grupo.

Otra premisa relevante en las reivindicaciones secesionistas sería la cuestión territorial. El territorio del grupo es necesario para alcanzar el fin último del proceso y desligarse de la autoridad central para establecer la propia. Uno de los objetivos es

\footnotetext{
${ }^{32}$ Martínez, A. (2015): óp. cit. p. 167.

${ }^{33}$ Buchanan, A. (2013): Secesión: causas y consecuencias del divorcio político, Barcelona: Ariel, p. 50.

${ }^{34}$ Ibíd., p. 50.
} 
conservar las fronteras nacionalistas existentes y salir del ámbito interno, trazar nuevas fronteras a partir de las propias para alcanzar la soberanía. ${ }^{35}$

Fijados algunos objetivos del proceso secesionista hay que aclarar que la secesión no es un derecho que pueda ejercerse libremente si se cumplen una serie de requisitos, sino que sólo puede llevarse a cabo si viene permitida en el texto constitucional de cada estado. Realizarla de cualquier otra forma, en principio, entraría bajo los supuestos de secesión unilateral ilegal. Por lo tanto, la secesión unilateral como ruptura con el orden interno vigente será asunto del derecho constitucional, diferente del derecho de autodeterminación, o en casos más graves la doctrina de la secesión como remedio, temas que se tratan desde la óptica internacional. Al confluir ambos términos es necesario una oportuna distinción, para justificar en cierta medida que el contexto internacional sea relevante en un asunto del que se ocupa el derecho interno de cada estado. ${ }^{36}$

Como se ha expuesto, los procesos de autodeterminación necesitan internacionalizarse para que la comunidad internacional se fije en ellos. Solo los conflictos territoriales que se vuelven relevantes a nivel internacional son considerados por la comunidad internacional para abordar en sus territorios el principio de autodeterminación. Sin embargo, parece que la secesión es todo lo contrario. Esta versará sobre aquellos casos en los que una disputa territorial no ha adquirido aún relevancia internacional y debe tratarse siempre conforme al derecho interno. En principio, la secesión es un asunto en el que el derecho internacional no está involucrado, y que generalmente se rige exclusivamente por el derecho constitucional. ${ }^{37}$

No quiere decir que la autodeterminación y la secesión no sean asuntos relacionados, ni que los diferentes órdenes legales que los rigen estén completamente separados. Es innegable que la comunidad internacional está preocupada por el gran número de intentos secesionistas tanto en las democracias ya asentadas y establecidas, como en aquellas zonas donde la democracia no es la forma de organización del estado. De hecho, un proceso secesionista puede, en cierto punto, ser internacionalizado. ${ }^{38}$ Los intentos desde la comunidad nacionalista que quiere lograr esta internacionalización para que el conflicto goce de una atención y protección distinta al conflicto interno son diferentes y cada grupo en su región lo ha acercado desde diferentes perspectivas. ${ }^{39}$ Del mismo modo las circunstancias que pueden llevar a tomar la secesión como un problema aceptable y relevante para la comunidad internacional son de naturaleza variada. Hay algunos casos en los que la comunidad internacional da pasos hacia el reconocimiento como estado a una región que aspira y traza esta dirección; o en otros reivindica y apoya el uso de la fuerza por parte de terceros involucrados en un conflicto de secesión. ${ }^{40}$

En la totalidad de las ocasiones, la intervención internacional ha tenido en la protección de los derechos humanos, aparente o real, el fundamento último para justificar su intervención y trasladar un problema territorial interno que debería ser resuelto por el marco constitucional vigente a la esfera internacional, respondiendo con los mecanismos internacionales que en ese caso concreto considere sean de aplicación.

\footnotetext{
${ }^{35}$ Ibíd., p. 51.

${ }^{36}$ Sunstein, C. (1991): “Constitutionalism and Secession”, University of Chicago Law Review, N. 58, pp. 633-670.

${ }^{37}$ Rodríguez, Á. (2019): "Moving towards independence - breach ing the law: self-determination and secession in Catalonia", Rassegna di diritto pubblico europeo, Vol. XVII, pp. 343-372.

${ }^{38}$ Ibíd., p. 360.

${ }^{39}$ Martínez, A. (2015): óp. cit., pp. 199-264.

${ }^{40}$ Rodríguez, Á. (2019): óp. cit., p. 361.
} 
La protección de los derechos humanos como el nexo más importante entre la preocupación de la comunidad internacional por respetarlos y reconocer nuevos estados. Esto justifica el derecho de autodeterminación de una minoría territorial dentro de un estado después de una violación sistemática y generalizada de los derechos civiles y humanos. En un principio la comunidad agredida inicia un proceso de secesión unilateral que podrá ser llevada a cabo y considerada un caso de secesión como remedio.

Esta secesión se transformará en un asunto internacional ya que implica en sus últimas consecuencias el nacimiento de un nuevo estado independiente. Para ello, el derecho internacional aplica estándares reconocidos para distinguir entre secesiones legítimas e ilegítimas, ya que un acto de secesión solo sería válido en virtud del derecho internacional si se basa en una reivindicación legítima del derecho de autodeterminación recogido en los textos internacionales, o en algunas ocasiones procedente de un caso excepcional considerado por la doctrina como un caso de secesión como remedio. ${ }^{41}$

Solo se admiten desde el derecho internacional estos casos de autodeterminación tras vulneraciones suficientes y repetidas a los derechos humanos. Habrá que dejar fuera de este marco aquellos ejemplos que no se adecuen al esquema. Así, todos los procesos secesionistas que se sigan en territorios no coloniales, distintos de los justificados por violaciones graves de los derechos humanos, están únicos y exclusivamente bajo la jurisdicción del derecho interno, y por lo tanto no implican el ejercicio de ningún derecho otorgado por el derecho internacional. ${ }^{42}$ Aunque, la secesión y la autodeterminación se encuentren unidas en la delgada frontera del derecho constitucional e internacional, a pesar de sus complejas relaciones ligadas se supone que la distinción entre la autodeterminación y la secesión es útil para el fin de este artículo, ya que se pueden acordar casos de secesión alejado de los límites del derecho internacional, al no ser el conflicto interno atractivo para esta esfera, no incluyéndolo como un asunto de autodeterminación. Esta afirmación nos acerca a la Opinión consultiva de la Corte Internacional de Justicia, cuando no trata la DUI en cierto modo por ser un aspecto político interno de cada estado, y no se acerca desde la doctrina internacional, aunque concluye así por otros razonamientos como he mostrado.

Así, se debe mantener esta distinción ya que se aplican criterios legales distintos en cada caso. Se puede suponer que una minoría territorial no tiene derecho a la autodeterminación desde el derecho internacional, al no encontrarse amparado por sus reglas, pero está sujeta a un hipotético derecho de secesión si así se recoge en el marco constitucional interno. En ese caso se inicia un proceso secesionista legítimo, que podría en algún momento ganar un reconocimiento internacional en virtud de la aceptación de este marco interno, una situación no muy frecuente. Por el contrario, se puede alcanzar la suficiente atención internacional que considere una secesión ilegítima como el ejercicio protegido del derecho de autodeterminación siempre y cuando entre bajo los parámetros internacionales, ya que una vulneración del ordenamiento jurídico interno implicaría una acción de ruptura con el marco constitucional vigente que podría, obtener reconocimiento internacional más adelante, desvinculado con la esfera interna del principio. Sin embargo, en esos casos se mantendrá el hecho de que aconteció una secesión ilegal desde un punto de vista interno.

Entendida la distinción es necesario mencionar uno de los mecanismos más relevantes que inicia una secesión ilegal. Este será la Declaración Unilateral de Independencia. Una

\footnotetext{
${ }^{41}$ Ibíd., p. 362.

${ }^{42}$ Rodríguez, Á. (2019): óp. cit., p. 363.
} 
acción que viene precedida del rechazo al orden constitucional y de una crisis política. La DUI se encuentra en el límite de la secesión, siendo considerada como una rebelión en sí misma, dado su carácter unilateral que omite el contexto constitucional. Esta situación produce conflicto social, tensión política e incluso la intervención militar. ${ }^{43}$ Este acto político de carácter separatista que pretende considerar propias las instituciones constitucionales y configurarlas para su proceso secesionista conllevaría un trastorno social y económico significativo, lo que aumentaría el riesgo de una desobediencia civil, violenta en la mayoría de las ocasiones. Una crisis de secesión trae una complejidad social y económica significativa, que va más allá de cualquier cuestión en apariencia relevante como la creación de nuevas fronteras, sino que se acerca a la creación de un nuevo orden jurídico. Construir una nueva soberanía desde la ruptura unilateral y total con el sistema vigente a través de una nueva política significa quebrantar el sistema constitucional. ${ }^{44} \mathrm{Y}$ romper con este marco interno vigente acarrea una serie de consecuencias desconocidas para los grupos secesionistas.

Para comprender el alcance real de la DUI y comprobar si llevarla a cabo se justifica en un caso extremo de vulneración de los derechos y libertades de las minorías, presento el caso de Kosovo y el caso de Cataluña, contextos donde se han efectuado sendas DUI y por ello resultan situaciones claves en las que se puede pensar si aplicar o no la secesión como remedio. Para ello haré un repaso breve de los antecedentes lógicos al efectuar la DUI. Posteriormente trato de encajar ambos casos bajo la doctrina de la secesión como remedio. Para ello se alude a los razonamientos jurídicos amparados en la doctrina internacional y se plantea si más bien estas conclusiones no enmascaran razones políticas que arbitrariamente utilizan determinados casos para presentarlos como ejemplos de secesiones legítimas, cuando haya motivos para considerar que no lo sean.

\subsection{Caso Kosovo desde la secesión como remedio.}

Tal y como he expuesto si el proceso secesionista de Kosovo comienza con la DUI tendríamos que encuadrarlo bajo el término de una secesión ilegal, al no contemplar la posibilidad de independencia unilateral el marco interno serbio, ni la Resolución 1244 (1999) del Consejo de Seguridad. Entonces, si es una cuestión que debe resolver el estado desde la Constitución, ¿por qué se ha categorizado como un problema de derecho internacional?

El conflicto de la minoría albano kosovar con Serbia, enquistado en el tiempo, y las vulneraciones graves y sistemáticas de los derechos humanos hacia un grupo atrae toda la atención internacional, incluyendo su implicación directa. Esta intervención en el gobierno serbio modifica el marco interno de manera ininterrumpida, intentando una solución pacífica en la que los actores principales se encuentren implicados. Tras ocho años de gobierno provisional por la Resolución internacional del Consejo de Seguridad, la Asamblea de Kosovo proclama su independencia unilateral iniciando un extraño proceso secesionista, ya que su gobierno provisional procede de un marco internacional. Esto pone de manifiesto la inestabilidad del gobierno, que buscaba la DUI como última salida aceptable al conflicto albano kosovar. Siendo un gobierno central débil, más posibilidades tenía el grupo secesionista de lograr el éxito de su independencia. Además, esta fragilidad del gobierno se vio respaldada desde una administración internacional,

\footnotetext{
${ }^{43}$ Haljan, D. (2014): Constitutionalising secession, Oxford: Hart Publications, p. 10.

${ }^{44}$ Ibíd., p. 11.
} 
para paliar los excesos represores y la falta de autoridad del gobierno central involucrando así a toda la comunidad internacional.

En primer lugar, para aceptar la DUI de Kosovo debemos situarnos bajo las coordenadas de un gobierno central débil sin legitimidad alguna para el pueblo kosovar ya que en realidad se haya bajo una administración internacional, como un paso relevante para que sea aceptable aplicar la doctrina internacional de la secesión como remedio. Así, cuanto más inestable es un gobierno central, mayor es la probabilidad de éxito del derecho de autodeterminación por parte de una región secesionista. Se debe demostrar, al no proceder de un contexto de descolonización, que se ha necesitado una administración internacional de su región en los últimos años, y que las autoridades internacionales han tenido que gobernar debido a la brutalidad e ineficacia del gobierno central hacia una minoría. $^{45}$

Bajo estas dos premisas, tres de los magistrados ${ }^{46}$ que participan en el dictamen consultivo de la CIJ comprenden que Kosovo es un caso tipo de secesión como remedio, al ser la salida más favorable para el grupo la secesión unilateral, al peligrar gravemente sus derechos humanos y civiles. Estas manifestaciones claras a favor de presentar Kosovo como un caso de secesión como remedio se recogen en las siguientes opiniones separadas. Así, en opinión separada del Magistrado Simma, aunque la Corte no se pronuncie sobre el aspecto de la autodeterminación, considera que los kosovares como actores de la DUI hicieron referencia al principio de libre determinación fundado en las vulneraciones de los derechos humanos, lo que les otorga la protección de la doctrina internacional para llevar a cabo la secesión como remedio como grupo. ${ }^{47}$

Del mismo modo en la opinión separada del Magistrado Cançado Trindade la declaración de independencia de Kosovo solo se puede considerar de forma apropiada analizada bajo la lupa de los complejos y trágicos antecedentes de hecho de la grave crisis humanitaria de Kosovo, que culmina con la aprobación de la Resolución 1244 (1999). Nos dice que por ello el derecho internacional se compromete con la calidad de vida de parte de la población y pone fin a la represión brutal que sufría el pueblo kosovar. ${ }^{48} \mathrm{La}$ crisis interna se internacionaliza y la intervención externa cobra sentido, siendo un caso de secesión como remedio.

También en la opinión separada del Magistrado Yusuf hay un derecho afirmativo que permite legitimar esta secesión unilateral, desde una concepción poscolonial. La violación de los derechos humanos supone abrir la puerta a la intervención internacional y saltarse el marco interno. Si las circunstancias excepcionales del caso examinado lo permiten, el grupo que declara la independencia unilateral puede ser respaldado por el derecho internacional, y la pretensión de autodeterminación así defendida ser válida y legítima. ${ }^{49}$

Además de la opinión separada de estos tres magistrados que participaron en la Opinión consultiva, y que responden desde una perspectiva que la CIJ deja al margen, tal

\footnotetext{
${ }^{45}$ Sterio, M. (2013): The right to self-determination under international law: "selfitans, secession, and the rule of the great powers", London: Routledge, p. 61.

${ }^{46}$ Votaron a favor de la no prohibición de la DUI de Kosovo desde el Derecho Internacional.

47 Summaries of Judgments, Advisory Opinions and Orders of the International Court of Justice. 180. Accordance with International Law of the Unilateral Declaration of Independence in respect of Kosovo. Advisory opinion of 22 July 2010, óp. cit., p. 23.

${ }^{48}$ Ibíd., p. 33.

${ }^{49}$ Ibíd., p. 38.
} 
y como comentamos anteriormente, la mayoría de la doctrina también confirma la DUI de Kosovo legítima bajo el prisma de la secesión como remedio, apoyándose en el dictamen de la CIJ de manera incompleta.

Afirman que en casos como el de Kosovo la comunidad internacional entiende que los crímenes contra la población albanesa en dicha región habían desembocado en una situación irreconciliable. Como última solución, la que finalmente se adoptó fue admitir la secesión unilateral como forma de garantizar la protección a los derechos humanos, y así resolver una situación que no encontraba otra salida. Para ello, se materializa el derecho de autodeterminación de ese grupo de la única forma posible que pueden, mediante la secesión unilateral a través de la DUI, dado que la integración con una autonomía en Serbia no era posible, y el marco interno no permitía la secesión pactada. ${ }^{50}$

Por tanto, la doctrina se apoya en las líneas marcadas por la CIJ (que no traza de ningún modo) en su Opinión consultiva sobre Kosovo, y reafirman que no existe una norma de derecho internacional que impida practicar casos de secesión como remedio, y que serán los estados los que establecerán mediante la práctica la secesión como remedio en la comunidad internacional. ${ }^{51}$

Esta conclusión, basada en el conjunto vacío que deja la Corte cuando omite relacionar Kosovo con la secesión como remedio es, cuando menos, equívoca, ${ }^{52}$ ya que otorga la decisión a la comunidad internacional de considerar si una secesión unilateral recae bajo la secesión como remedio y no se aplica el marco interno. Con la excusa de que la Opinión consultiva traza una línea afirmativa en defensa de los derechos humanos, en realidad el dictamen de la CIJ no se manifiesta en este sentido, permite la secesión unilateral en casos extremos y la justifica con la complacencia de la comunidad internacional.

Pero ¿sería posible reivindicar la vulneración de los derechos de la minoría albanesa cuando estaba bajo un gobierno provisional estable, que iba a producir un cambio de régimen tras celebrar unas elecciones democráticas? Tras ocho años, donde hubo importantes avances en el diálogo con el nuevo gobierno serbio, que era eficiente y estaba dispuesto a otorgar distintas concesiones a la región albanesa de acuerdo con el marco interno, parece difícil justificar esta reivindicación. En el momento en el que se declaró la independencia, no se podía argumentar que era necesario detener los actos de opresión. Puede ser que el recuerdo de la represión brutal excluyese la posibilidad de una nueva autonomía para Kosovo y la restauración pacífica de la autoridad serbia ${ }^{53}$, pero esto parece más una cuestión política que una defensa a ultranza de las minorías.

Incluso si hubo represión durante estos años, justo antes de la secesión unilateral algunos estados no consideraban que la respuesta desde la comunidad internacional fuese fomentar el castigo a la región opresora en forma de conceder una autonomía no contemplada en el marco interno y la consecuente pérdida de territorio. ${ }^{54}$

El caso Kosovo defendido desde la secesión como remedio hace preguntarse qué se entendió por violación grave de los derechos humanos y cuál fue el periodo de tiempo

\footnotetext{
${ }^{50}$ Martínez, A. (2015): óp. cit., p. 276.

${ }^{51}$ Martínez, A. (2015): óp. cit., p. 278.

52 Sanz, C. (2017): El derecho de autodeterminación: constitución y normas internacionales. Navarra:

Thomson Reuters Aranzadi, p.247.

53 Summers, J. (2014): “Kosovo" en Walter, C., Von Ungern-Sternberg A., Abushov, K., (ed.): Selfdetermination and secession in international law, Oxford: Oxford University Press, p. 251.

${ }^{54}$ Ibíd., p. 252.
} 
considerable bajo el que se ejerció esta opresión y por parte de quién. Además, es problemático considerar que la secesión unilateral era la única solución al problema, si los principales actores se estaban encaminando hacia un final que no consistiera en la ruptura total con el marco constitucional. Estas dudas suscitaron la mayor parte de desacuerdos entre estados que reconocieron a Kosovo como región soberana e independiente, que se apoyan en una doctrina internacional que no parece encajar del todo en este caso. ${ }^{55}$

Por todo esto, hay autores que no comparten esta secesión unilateral y presentan unas motivaciones políticas, más que jurídicas, como las verdaderas razones para aceptar el caso Kosovo. Así, resultó grave plantear una secesión unilateral en 2008 contra un régimen democrático en Serbia, respetuoso con los derechos de todos los ciudadanos, $\mathrm{y}$ consciente y preparado para dotar a Kosovo de la más extensa autonomía. ${ }^{56}$ El precedente de Kosovo que rompe la integridad territorial serbia y reorganiza sus fronteras desde un grupo minoritario es aceptado por la mayoría de los estados de la comunidad internacional, y la única razón posible es comprender este hecho bajo una represión a las minorías en el pasado, encuadrándose no bajo el caso de la secesión como remedio, sino bajo el contexto de la secesión como castigo.

Además, la secesión como castigo no ha sido la práctica de la comunidad internacional en otros estados en los que había aspiraciones secesionistas y violaciones flagrantes de derechos humanos. Por ejemplo, no fue el caso en lugares como Macedonia o Bosnia-Herzegovina, tampoco fomentar la secesión fue la respuesta de la comunidad internacional, como en Timor Oriental, sino que se intentan establecer procesos de diálogo entre las partes que contemplen una solución pacífica, como Etiopía-Eritrea. En la mayoría de los casos, como en el Tíbet, Palestina o Sáhara Occidental ni siquiera se busca una solución rápida, sino que son conflictos que permanecen en el tiempo. Por último, en la mayoría de las ocasiones se ha intentado defender la integridad territorial y la autoridad central para evitar el reconocimiento de nuevos estados, como en los casos de Abjasia y Osetia del Sur, que respaldaron en un primer momento el marco interno de Georgia. ${ }^{57}$

Tras esta mención a casos tan diversos se ve como la DUI nunca fue la solución, menos aún una independencia aceptada por la CIJ y por la comunidad internacional. La secesión unilateral no ha sido acogida como la mejor respuesta a casos de violaciones de derechos humanos o a una falta de acuerdo entre regiones y la autoridad central en los casos mencionados. Además, en el caso que he expuesto, Kosovo no era una colonia y estaba gobernada por una autoridad provisional que estaba buscando una respuesta estable a un problema regional, por lo que la represión no tenía lugar en el momento de la DUI. Entonces, ¿por qué el afán de presentar Kosovo como un caso tipo de secesión legítima bajo la doctrina internacional? Si bien la CIJ no se pronuncia sobre el caso Kosovo en estos términos, ¿por qué interesa encajarlo en este contexto?

Para Milena Sterio hay una respuesta política clara a estas cuestiones defendidas desde razonamientos jurídicos. Para ella la verdadera razón en aceptar Kosovo como estado soberano está en lo que define como "The Rule of the Great Powers". 58 Si las

\footnotetext{
${ }^{55}$ Ibíd., p. 253.

${ }^{56}$ Mangas, A. (2011): "The European Union and Kosovo: A Planned Secession”, Revista Española de Derecho Internacional, Núm. 63, p. 109.

${ }^{57}$ Mangas, A. (2011): óp. cit., p. 110.

${ }^{58}$ Sterio, M. (2013): óp. cit. pp. 57-72.
} 
grandes potencias, los estados concebidos por la comunidad internacional como más importantes están de acuerdo en que Kosovo debe ser independiente, entonces se argumenta que la secesión unilateral es una buena solución y el final más correcto. ${ }^{59}$

En este apartado se demuestra que Kosovo no es un ejemplo claro de secesión legítima. Siendo coherentes opino que es un caso excepcional y especial ${ }^{60}$ por las razones mencionadas y la verdadera causa para ubicarlo en un caso tipo que ejerce el derecho de autodeterminación, cuando la CIJ no se manifiesta a favor en este sentido, serán unas razones políticas que desconozco pero que tratan de organizar la comunidad internacional en función de unos intereses.

Una vez considero que la secesión unilateral de Kosovo no puede ampararse bajo la secesión como remedio, examino el caso de Cataluña a partir de la DUI. Se comprueba si no puede encajar en esta doctrina, o, por el contrario, con otra interpretación podría la DUI ser el inicio de un proceso secesionista legítimo con repercusiones en la comunidad internacional.

\subsection{Caso Cataluña desde la secesión como remedio.}

En primer lugar, siguiendo con el principio de autodeterminación expuesto hay que declarar que Cataluña no es una colonia de España, ni nunca lo ha sido. Por lo que no se puede aplicar el derecho de autodeterminación en un contexto de descolonización. ${ }^{61}$

Si descartamos este primer supuesto hay que continuar examinando los distintos escenarios en los que se podría considerar una secesión legítima. En segundo lugar, en el caso estudiado se establece que declaran la independencia unilateralmente lo que encamina al grupo nacionalista catalán a un proceso secesionista ilegítimo.

Además, en dicha declaración se pretende tener como modelo la DUI albano kosovar, con la que buscan un reconocimiento internacional que no impida esta secesión unilateral, sino que sea aceptable y aceptada, ya ofreciendo como precedente el dictamen de la CIJ, lo que les otorgaría en teoría una posición beneficiosa. Los autores que apoyan la secesión unilateral consideran la DUI como el paso necesario para poner de manifiesto que el caso catalán sigue unas pautas dictadas por la comunidad internacional, que pudiera respaldarles en la defensa de su opción secesionista. ${ }^{62}$

Se intenta a través de la DUI que la independencia de Cataluña sea favorable bajo el supuesto de la secesión como remedio. Analizando los sucesos ocurridos los días que precedieron a la DUI y la situación de Cataluña no se puede concluir que los derechos civiles de la minoría catalana estaban siendo vulnerados ${ }^{63}$, ni que la autoridad del estado español estaba actuando de manera brutal o represiva sistemáticamente durante un

\footnotetext{
${ }^{59}$ Ibíd., p. 69.

60 Vidmar, J. (2012): "Conceptualizing Declarations of Independence in International Law." Oxford Journal of Legal Studies, Vol. 32, Núm. 1, pp. 153-177.

${ }^{61}$ Elliot, J. (2018): Catalanes y escoceses: unión y discordia. Barcelona: Taurus.

${ }^{62}$ Bossacoma, P. (2015): Justícia i legalitat de la secessió. Una teoria de l'autodeterminació nacional de Catalunya, Barcelona: Institut d'Estudis Autonòmics, pp. 236-240.

${ }^{63}$ Torroja, H. (2019): The self-determination of peoples vs human rights in liberal democracies: the case of Catalonia, epígrafe 2.3, párrafo cuarto, disponible en :

http://www.realinstitutoelcano.org/wps/portal/rielcano_en/contenido?WCM_GLOBAL_CONTEXT=/elc ano/elcano_in/zonas_in/ari99-2019-torroja-self-determination-of-peoples-vs-human-rights-in-liberaldemocracies-the-case-of-catalonia [consultado el 9 de enero de 2020]
} 
periodo de tiempo considerable. ${ }^{64}$ Los requisitos aceptados por la comunidad internacional para considerar el caso catalán dentro de una violación de los derechos humanos de las minorías tampoco pueden cumplirse efectivamente, al margen de la propaganda.

Los intentos de exponer esta falta de respeto hacia los derechos fundamentales del grupo catalán que tengan trascendencia en el marco internacional han sido numerosos. La mayoría están contenidos en el relato del agravio. Esto iría encaminado a que la comunidad internacional haga relevante un problema interno e intervenga. Como se expuso anteriormente la internacionalización de la secesión junto a cuestionar la autoridad central, presentando a un gobierno débil, son pasos relevantes para forjar un proceso secesionista legítimo, que tenga cabida en la doctrina de la secesión como remedio.

Se puede creer que no es necesario aludir a una violencia extrema contra las minorías, si el recuerdo de esta en otros casos ha bastado para aceptar la secesión unilateral. Se intenta a través de distintos contextos, como la lengua, la democracia, la educación, la justicia o la economía fabricar un relato coherente y creíble que induzca a pensar a la comunidad internacional que los derechos humanos de un grupo están siendo vulnerados, por lo que no deberían respetar el marco constitucional del estado opresor. Dado que no están inmersos en un conflicto bélico de la envergadura de Kosovo, ${ }^{65}$ se crean utilizando los medios a su alcance situaciones extremas que hagan necesaria la presencia internacional, lo que en el primer apartado se configura como el relato del agravio consolidado a través del procés.

En principio, no es posible conocer qué nivel de afrenta debe alcanzar la vulneración del derecho para que se fije en él la comunidad internacional. En última instancia, serían los "grandes poderes" los que decidieran que solución política otorgarle al conflicto territorial, ya que no atenderían a los razonamientos jurídicos existentes. Por lo que sería impredecible ponderar que estos sucesos, en apariencia irrelevantes comparados con otros casos, encajaran conforme a una interpretación adecuada en la secesión como remedio. Así que cualquier argumento que manifieste que hay una minoría oprimida en Cataluña por una autoridad central que no reconoce su autonomía será bienvenido para el procés, y fomentaría que una secesión unilateral pueda alcanzar dimensión internacional.

Dentro de esta vulneración de derechos el nacionalismo independentista catalán ha incidido en la teoría del expolio, ${ }^{66}$ la vertiente económica del procés, bajo la premisa "Espanya ens roba". Se repite en numerosas ocasiones la cuestión económica como agravio comparativo y vulneración de su autonomía esgrimiendo cifras ${ }^{67}$ que han sido compartidas en organismos internacionales. Al margen de que la cuestión pueda resultar irrisoria $^{68} \mathrm{o}$ improcedente si estamos buscando elementos que hagan factible la secesión como remedio desde la DUI, no estaría de más recordar que la diferencia económica entre regiones y la justicia distributiva está contemplada por Allen Buchanan como causa para

\footnotetext{
${ }^{64}$ Arenas, R. (2018): “El procés, un intento de secesión de hecho”, en Coll, J., Molina, I. y Maldonado, M. (ed.): Anatomía del procés. Barcelona: Debate, pp. 67-88.

${ }^{65}$ Bassets, L. (2018): "Los maestros narradores del independentismo”, óp. cit., pp. 159-179.

${ }^{66}$ Llorach, J. (2018): "El relato del expolio: el triunfo de Junqueras", óp. cit., pp. 89-116.

${ }^{67}$ VV.AA. (2013): Claves sobre la independencia de Cataluña, Barcelona: Editorial Comanegra, pp. 7190; Vidal-Folch, X. (2013): ¿Cataluña independiente?, Madrid: Los Libros de la Catarata, pp.60-67.

${ }^{68}$ Borrel, J. y Llorach, J. (2015): Las cuentas y los cuentos de la independencia, Madrid: Los Libros De La Catarata.
} 
la secesión, aunque no la considera legítima. ${ }^{69} \mathrm{El}$ argumento de la redistribución de la riqueza entre las distintas regiones como agravio que minusvalore derechos también se ha defendido por parte de otras regiones europeas, como el caso de Baviera en Alemania o Véneto en Italia. ${ }^{70}$ Es importante reseñar que la cuestión económica ha sido importante a lo largo del procés, desde 2010, y su pretendida financiación declarada inconstitucional por el TC, por lo que este punto es un argumento fuerte desde el nacionalismo catalán para expresar una represión por parte de la autoridad central que no les permita un pleno desarrollo económico. Dentro de la lógica secesionista este agravio fiscal es otro elemento que les hace meritorios de encajar su caso bajo la secesión como remedio.

Repasando el caso catalán se concluye que no encaja bajo la doctrina internacional de la secesión como remedio, por lo que la pretendida ruptura con el marco constitucional al considerar que no posee autoridad sobre el grupo no sería posible. Entiendo que presentar la DUI como gran paso para acercar el conflicto a la comunidad internacional y exponer la vulneración de derechos civiles de la minoría catalana por parte del Estado español no da el resultado esperado. Este sería transformar la secesión unilateral en un proceso secesionista legítimo. Dado que no se reconoce ni desde el marco constitucional interno ni desde la vertiente internacional habría que esperar a la solución política que recibe el procés.

\section{Conclusiones.}

A lo largo de estas páginas se han presentado situaciones parecidas que intentaron romper el orden constitucional vigente, aunque con distinto resultado. La proclamación de la DUI por parte de la Asamblea de Kosovo goza del respaldo internacional al recordar la vulneración de los derechos humanos que sufrió su población por parte de Serbia. Que este argumento no sea validado por la Corte para defender que la DUI no estaba prohibida por el derecho internacional no le resta importancia. No pronunciarse sobre lo que significa una grave y reiterada violación de los derechos de las minorías que permita la secesión como remedio abre la puerta a que otros grupos nacionalistas tomen esta vía como referencia.

Así, la DUI del Parlament de Cataluña buscaba la secesión como remedio presentándose ante la comunidad internacional como una región oprimida por España, cuyos últimos actos, los sucesos del 1 de octubre se mostraron como verdaderas muestras de terror y represión por parte de la autoridad central. Esto da lugar a la culminación del procés y a una completa internalización de la secesión ilegitima de Cataluña al no contemplarse desde el derecho constitucional. Conseguir la secesión con el apoyo de la comunidad internacional era el fin último del procés. Si bien la DUI no ha sido efectiva ni ha contado con ningún apoyo, es otro hito en la revisión de la cuestión catalana y en la hoja de ruta nacionalista que quiere presentar un problema interno bajo el marco constitucional español como una cuestión internacional que incumbe en gran medida a Europa.

Que ambas DUI hayan sido comparadas y parecidas desde la identidad de sus autores y la forma en la que se efectuaron pone de relieve el modelo a seguir de los grupos nacionalistas catalanes, Kosovo, Eslovenia o Montenegro; regiones donde la violencia consiguió que el proceso de secesión captara la atención internacional, y se buscara una solución política al problema que implicara a múltiples sujetos. Esta solución política en

\footnotetext{
${ }^{69}$ Buchanan, A. (2013): óp. cit., pp. 189-203.

${ }^{70}$ Beltrán, M. (2019): Myths and Realities of Secessionisms.A Constitutional Approach to the Catalonian Crisis, Suiza: Palgrave Macmillan, pp. 60-62.
} 
la que intervengan otros estados es lo que se intentó con la DUI catalana. La respuesta política es la única que puede esperar el independentismo catalán ya sea por parte de España o de la comunidad internacional. Como se ha puesto de manifiesto en este artículo, su proyecto de secesión legítima a partir de la DUI no está amparado por el derecho interno español ni por el derecho internacional.

\section{Bibliografía.}

- BELTRÁN, M. (2019): Myths and Realities of Secessionisms.A Constitutional Approach to the Catalonian Crisis. Suiza: Palgrave Macmillan.

- BORREL, J. y LLORACH, J. (2015): Las cuentas y los cuentos de la independencia. Madrid: Los Libros De La Catarata.

- BOSSACOMA, P. (2015): Justícia i legalitat de la secessió. Una teoria de l'autodeterminació nacional des de Catalunya. Barcelona: Institut d'Estudis Autonòmics,

- BOSSACOMA, P. (2017): Secesión e integración en la Unión Europea. Cataluña ¿nuevo Estado de la Unión? Barcelona: Generalitat de Catalunya. Institut d'Estudis d'l Autogovern.

- BUCHANAN, A. (2013): Secesión: causas y consecuencias del divorcio político. Barcelona: Ariel.

- BUCHHEIT, L.C. (1978): Secession: the legitimacy of self-determination. New Haven: Yale University Press.

- CARDONA, J. (2011): "La relevancia (o no) de la administración internacional de Kosovo en la opinión consultiva de la Corte Internacional de Justicia de 22 de julio de 2010: perplejidades de un lector," Revista Española de Derecho Internacional, Núm. 63, pp. 79-100.

- COLL, J., MOLINA, I. y ARIAS, M. (ed.) (2018): Anatomía del procés. Barcelona: Debate.

- DE MIGUEL, J. (2016): "Manual para construir un estado (Los informes del Consejo Asesor para la Transición Nacional de Cataluña)", Teoría y Realidad Constitucional, Núm. 37, pp. 499-527.

- ELLIOTT, J. (2018): Catalanes y escoceses: unión y discordia. Barcelona: Taurus.

- HALJAN, D. (2014): Constitutionalising secession. Oxford: Hart Publications.

- KNOP, K. (2002): Diversity and Self-Determination in International Law. Cambridge: Cambridge University Press. 
- LÓPEZ-BASAGUREN, A. (2016): "Demanda de secesión en Cataluña y sistema democrático. El procés a la luz de la experiencia comparada". Teoría y Realidad Constitucional, Núm. 37, pp. 163-185.

- MANGAS, A. (2011): "The European Union and Kosovo: A Planned Secession", Revista Española de Derecho Internacional, Núm. 63, pp. 101-123.

- MARTÍNEZ, A. (2015): El derecho de autodeterminación de los pueblos en el siglo XXI. Navarra: Thomson Reuters Aranzadi.

- RODRÍGUEZ, Á. (2019): "Moving towards independence - breach ing the law: selfdetermination and secession in Catalonia", Rassegna di diritto pubblico europeo, Vol. XVII, pp. 343-372.

- SANZ, C. (2017): El derecho de autodeterminación: constitución y normas internacionales. Navarra: Thomson Reuters Aranzadi.

- SOROETA, J. (2018) "Quo Vadis Cataluña? La unilateralidad, un viaje de corto recorrido", Anuario Español de Derecho Internacional, Núm. 34, pp. 323-342.

- STERIO, M. (2013): The right to self-determination under international law: "selfitans, secession, and the rule of the great Powers." London: Routledge.

- SUNSTEIN, C. (1991): "Constitutionalism and Secession." Chicago: University of Chicago Law Review, Núm. 58, pp. 633-670.

- TORROJA, H. (2019): The self-determination of peoples vs human rights in liberal democracies: the case of Catalonia, disponible en:

http://www.realinstitutoelcano.org/wps/portal/rielcano_en/contenido?WCM_GLOB AL_CONTEXT=/elcano/elcano_in/zonas_in/ari99-2019-torroja-self-determinationof-peoples-vs-human-rights-in-liberal-democracies-the-case-of-catalonia

- V.V. A.A. (2014): Libro Blanco de la Transición de Cataluña. Barcelona: Generalitat Catalunya. Departamento de Presidencia.

- VV.AA. (2013): Claves sobre la independencia de Cataluña. Barcelona: Editorial Comanegra.

- VIDAL-FOLCH, X. (2013): ¿Cataluña independiente? Madrid: Los Libros De La Catarata.

- VIDMAR, J. (2011): “The Kosovo Advisory Opinion Scrutinized”, Leiden Journal of International Law, Núm. 24, pp. 355-383.

- VIDMAR, J. (2012): "Conceptualizing Declarations of Independence in International Law." Oxford Journal of Legal Studies, Vol. 32, Núm. 1, pp. 153-177. 
- WALTER, C., VON UNGERN-STERNBERG, A., ABUSHOV, K. (ed.) (2014): Self-determination and secession in international law. Oxford: Oxford University Press.

\section{Jurisprudencia.}

- International Court of Justice. Accordance with International Law of the Unilateral Declaration of Independence in Respect of Kosovo, Advisory Opinion, 2010 I.C.J. 404 (July 22). Disponible en:

https://www.icj-cij.org/files/case-related/141/141-20100722-ADV-01-00-EN.pdf

- Summaries of Judgments, Advisory Opinions and Orders of the International Court of Justice. 180. Accordance with International Law of the Unilateral Declaration of Independence in respect of Kosovo. Advisory opinion of 22 July 2010 